\title{
Misión del docente: propiciar en el estudiante aprendizajes significativos
}

\section{Teacher's mission: to promote meaningful learning's}

Mtra. Hortensia Murillo Pacheco•

\section{Resumen}

En este artículo se invita a los profesores a reflexionar sobre la práctica docente que realizan cotidianamente. Un aspecto nodal en el que pocas veces se repara es la forma en que se relaciona a los estudiantes con el conocimiento propio de cada asignatura. Este no es un asunto sencillo, se tendría que empezar con la revisión de la propuesta curricular en donde está(n) inserta(s) la(s) asignatura(s). Para conocer fundamentalmente el perfil del egresado, la orientación que tiene la formación de los estudiantes, qué tipo de necesidades sociales se pretende cubrir, etc. Es importante revisar y traer a la conciencia los conceptos que todo docente sustenta sobre aprendizaje, aprendizaje significativo, enseñanza y por ende también sobre objetivos de aprendizaje, contenidos temáticos y en particular sobre la metodología del proceso enseñanza-aprendizaje (que refleja justamente la forma de relacionar a los estudiantes con el conocimiento) y por último sobre acredita-

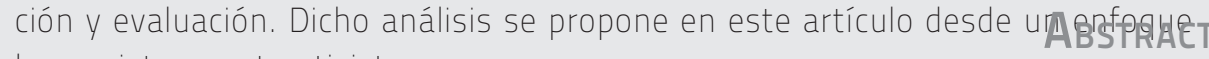
humanista-constructivista.

In this article, teachers are invited to think about their daily teaching practice. One main aspect, usually forgotten, is the way in which teachers relate students with knowledge of each subjetc. This is not an easy matter, we should begin with the review of the proposed curriculum where the subjects are inserted, in order to know, basically, the characteristics of graduated students, what sort of social needs are intended to be satisfy, etc. It is important to review and to be aware of concepts that any professor has about learning, meaningful learnig, teaching and therefore teaching objectives, syllabus and especially the methodology in the Teaching-learnig process (that reflects precisely the way in which teachers relate students with knowledge) and lastly grading and evaluation. This analysis is proposed in this article from a humanits-constructivist point of view.

-Asesora Pedagógica del Sistema de Universidad Abierta, ENEO-UNAM correspondencia: hortensia93@hotmail.com

RECIBIDO 29 JULIO 2010

EnVIAdo 3 AgOSTO 2010

ACEPTAdo 12 SEPTIEMBre 2010 


\section{INTRODUCCIÓN}

El título de este artículo constituye una verdad de Perogrullo. Sin embargo, a pesar de la obviedad, es necesario aclarar qué entendemos por "aprendizajes significativos".

En algunos casos, sobre todo cuando no se ha tenido una formación en aspectos psicopedagógicos, los docentes consideran que: basta con preparar la clase, exponerla lo más claro posible a los estudiantes, presentar algunos ejemplos o ejercicios, interrogarlos sobre algún aspecto del contenido, preguntarles si tienen dudas, dejarles algunos ejercicios, problemas o investigaciones para afirmar el conocimiento y al final de las unidades temáticas o bien del curso, aplicar exámenes para constatar qué tanto aprendieron.

La sorpresa es que los resultados no siempre son satisfactorios. Ante estos hechos, los docentes nos preguntamos por qué los alumnos no aprenden todo lo que nosotros quisiéramos, no obstante los enormes esfuerzos que realizamos.

Un aspecto que tendríamos que analizar es la forma en que relacionamos a los estudiantes con el conocimiento. En la situación anteriormente descrita la relación es mecánica, incluso pudiera ser un tanto ajena al estudiante, pues queda fuera de sus intereses, experiencias y necesidades. No se compromete al alumno ni intelectual ni emocionalmente, ni tampoco se ponen en juego todas sus capacidades cognitivas, obligándolo a que en ocasiones sólo se dedique a memorizar la información de los libros o los apuntes con la intención de obtener "buenas calificaciones" y acreditar la materia. Esa es la meta, el educando no pretende aprender, saber, manejar el conocimiento.
No obstante esta enajenación ante el aprendizaje, con frecuencia nos asombra y hasta nos enoja que los jóvenes, sobre todo de bachillerato o nivel superior, no sean capaces de ordenar sus ideas, de manejar un vocabulario amplio, de tener buena ortografía, de expresarse en forma oral y escrita con claridad y coherencia. Entonces, qué sucede, por qué tal mediocridad.

Existe la idea de que a los jóvenes no les importan estos aspectos, que están preocupados y ocupados en cosas supuestamente intrascendentes y triviales. Como educadores no podemos aceptar tal fatalismo. Más bien tendríamos que revisar cuál es nuestra responsabilidad frente a tales resultados. Los docentes no consideramos que los alumnos cuentan potencialmente con un cúmulo de capacidades y somos precisamente nosotros quienes deberíamos ayudar a desarrollarlas. La manera de hacerlo es justamente a través de la forma en que los conducimos en esa relación con el conocimiento de cada una de las disciplinas que conforman el currículo escolar.

En función de cómo guiemos a los estudiantes dependerá cómo se viva el proceso de aprendizaje: ya sea difícil y doloroso o bien todo lo contrario. Aprender, en el sentido de construir y descubrir, es una situación placentera que influye en la autoestima, en la seguridad de la persona, en su crecimiento, en su forma de entender, de interpretar el mundo y de llegar a establecer maneras distintas de relacionarse con el mundo y con las personas que le rodean.

Luego entonces, por qué este proceso en la escuela se torna tan tortuoso, difícil, estresante, etc., y en ocasiones provoca que los es- tudiantes la abandonen. En no pocas ocasiones, encontramos que jóvenes considerados inteligentes o muy inteligentes, obtienen resultados muy pobres durante su vida escolar. La escuela no tiene ningún atractivo, es un espacio inerte, que ofrece una relación autoritaria, fría y distante con los docentes y un cúmulo de contenidos sin sentido, carentes de interés para su edad, su contexto y sus necesidades.

\section{ANÁLISIS DE LA PRÁCTICA DOCENTE}

De ahí, la importancia de reflexionar sobre la forma en que realizamos nuestra práctica docente. La primera reflexión se refiere al hecho de entender que dicha práctica rebasa los límites del aula, que ésta se enmarca en un ámbito más amplio, que comprende las llamadas fuentes del currículum: la sociológica, la psicológica, la epistemológica y la didáctica. ${ }^{1}$ La primera tiene que ver con el propósito, la razón, y la justificación de la existencia de una propuesta curricular referida a un nivel escolar o a una profesión determinada. Es decir, hay que preguntarnos a qué tipo de necesidades e intereses sociales responde, qué concepciones ideológicas subyacen en dicha propuesta curricular, qué tipo de persona (sujeto) se pretende formar, etc. Obviamente la fuente sociológica está relacionada con la epistemológica. Esta última involucra el análisis del qué se va a enseñar, lo cual comprende desde la organización curricular (currículo abierto, cerrado, por asignaturas, áreas, módulos), objetivos de la carrera y de las asignaturas, tipo de contenidos propuestos y enfoques teóricos que subyacen, ya que todo esto va a influir en la 
orientación ideológica relacionada con la formación que recibirán los estudiantes.

En cuanto a las fuentes pedagógica y psicológica se encuentran estrechamente vinculadas, ya que la primera se refiere a cómo enseñar, es decir; qué estrategias de enseñanza y aprendizaje vamos a proponer en el desarrollo de las sesiones de clase. La segunda se refiere a entender cómo se dan los procesos de enseñanza-aprendizaje (enfoque teórico desde donde se hace la interpretación: conductismo, constructivismo, etc.), tomando en consideración edad, tipos de aprendizaje, nivel de maduración, etc., de los estudiantes.

Las fuentes sociológica y epistemológica escapan a nuestra participación, lo que comúnmente sucede cuando nos incorporamos a la docencia es que ya exista un currículum establecido. Si acaso lo que pudiéramos hacer es participar en su evaluación y/o restructuración o actualización de contenidos, pero no siempre es posible.

En cambio, en donde los docentes sí tenemos una responsabilidad directa, es en el análisis y manejo de las fuentes de enseñanza pedagógica y psicológica. Primero tenemos que conocer (si es que están explícitas), las concepciones que subyacen tras la propuesta curricular en la que está inserta la asignatura que vayamos a coordinar. Segundo, hay que investigar sobre dichas concepciones para intentar hacer una docencia profesional, con fundamentos teóricos

\section{Concepto de aprendizaje}

Lo analizaremos desde el enfoque constructivista. Éste postula que aprender significa construir esque- mas (conceptos) nuevos y reestructurar o ampliar los que ya existen (estructura cognitiva). No somos conscientes de todos los esquemas que poseemos en cada una de las etapas de nuestra vida y de que a partir de ellos construimos nuestras formas de pensar, sentir, actuar y relacionarnos. ${ }^{2}$

La construcción, ampliación o reestructuración de esquemas posibilita múltiples aprendizajes cada vez más complejos. Cada nuevo aprendizaje se construye a partir de la utilización de los esquemas o estructuras que ya se poseen. Es decir, las estructuras del conocimiento están en constante movilización o reestructuración.

Siguiendo al biólogo, psicólogo y epistemólogo suizo Jean Piaget, ${ }^{2}$ cada individuo va construyendo sus esquemas a partir de: a) el entorno físico y social b) la maduración, y c). la experiencia

a) Entorno físico y social. Todos los seres humanos nacemos en un lugar geográfico determinado y dentro de un grupo sociocultural con características propias a las que nos vamos incorporando paulatinamente. A través de la socialización se asimilan formas de vida, de pensamiento y de relación, lenguaje, religión, costumbres, mitos, valores, tradiciones, etc., y esto es lo que va conformando los esquemas y las estructuras de conocimiento de cada sujeto, en combinación con la maduración y la experiencia.

b) Maduración biológica: es el proceso de transformación del sistema nervioso que controla las capacidades intelectuales en potencia, lo que va a determinar los momentos oportunos para los aprendizajes (gatear, caminar, controlar los esfínteres, trepar, correr, hablar, leer, escribir, construir dimensiones de tiempo y espacio, etc.)

c) Experiencia. Los esquemas, y por tanto las estructuras de conocimiento que cada sujeto construye, son el resultado de sus propias acciones. Así cada persona crea su mundo y estas creaciones presentan diferencias tanto entre los individuos como entre las culturas (entorno físico y social.) Es decir, las experiencias que tenga una persona que vive en la ciudad serán diferentes a las que tenga una que vive en el campo, lo mismo que las que viven en la sierra, en la costa, o bien en el desierto, o en países orientales u occidentales. Sus formas de vida, de relación, de pensar, entre otras cosas, estarán de acuerdo a sus conocimientos, su filosofía, sus valores, tradiciones, costumbres, etc. Toda acción implica asimilar nuevos esquemas a los ya existentes, o bien reestructurarlos.

Las acciones se realizan en función de necesidades que van surgiendo. En la medida que se modifican o construyen esquemas se van modificando las acciones futuras.

Tomando como base este enfoque, el fin de la educación y del aprendizaje no es aumentar los conocimientos de las personas, sino modificar sus esquemas, sus estructuras 
cognitivas mediante la acción y la experiencia, en función de necesidades o metas que cada individuo se fija de acuerdo a su contexto específico. Es conveniente aclarar que las necesidades de cada persona no son estáticas, dependen de cada momento o etapa de la vida y de su entorno socioeconómico.

Ausubel, psicólogo norteamericano, retoma el concepto de aprendizaje como formación, reestructuración, enriquecimiento, etc. de esquemas y aporta otro elemento sustantivo para la construcción de dicho concepto que es el aprendizaje significativo. Éste consiste en relacionar los esquemas que toda persona posee, por su experiencia de vida, con los nuevos contenidos de cada materia. Gracias a la relación que se establece entre los conocimientos previos del alumno y el nuevo contenido, objeto de aprendizaje, los estudiantes construyen sentido y significado acerca de lo aprendido. Esto es, tanto el alumno como el grupo deben tener claridad sobre los objetivos planteados; la razón por la cual están trabajando conjuntamente, (lo que algunos autores llaman la tarea). Alrededor de ésta se van tejiendo una serie de vínculos con el conocimiento y entre los integrantes del grupo (interacción). Sólo que se dé dicha relación entre lo que ya conocen los educandos y los nuevos contenidos curriculares, se puede considerar que los sujetos realizan aprendizajes significativos, duraderos, que modifican sus esquemas previos, formando nuevas estructuras cognitivas, que se traducirán en cambios en su forma de pensar, sentir, actuar y relacionarse.

Los docentes debemos tener en cuenta que para promover aprendizajes significativos en los estudiantes se requieren las siguientes condiciones básicas, según Ausubel: ${ }^{3}$

1. Los nuevos contenidos que ofrece cada materia del Plan de estudios deben ser potencialmente significativos y sustantivos dentro del corpus de conocimientos del área correspondiente. Esta condición la denomina significatividad lógica.

2. En la estructura cognitiva del sujeto deben existir los esquemas previos necesarios que puedan ser relacionados con los nuevos contenidos. Esto constituye la significatividad psicológica.

3. Los estudiantes deben tener una actitud favorable para aprender significativamente, es decir; que estén motivados para ello.

\section{Concepto de enseñanza}

Desde la postura constructivista, el papel del educador no es el de transmisor de conocimientos. Es el que prepara y planea una serie de estrategias que promuevan la participación de los educandos. Esto es, conducirlos a revisar y confrontar sus ideas, conceptos, conocimientos previos, etc., relacionados con la materia y al mismo tiempo que ana-

- Se considera como tal el diseño de una sesión de clase, un tema o una unidad didáctica que contenga todas las actividades a desarrollar por el docente y por los estudiantes (individualmente y en equipo), así como los recursos o apoyos didácticos que se emplearán, para el logro de los objetivos de aprendizaje previamente especificados y en función de los contenidos.

licen, infieran, descubran, tomen conciencia a través de relacionarse con la información científica, que se les proporcione. Es decir, hacer que los estudiantes participen de alguna manera, que sean actores participantes, no meros espectadores de algo que no es significativo para ellos. El educando debe ser desafiado para asumir el papel de sujeto activo que se enfrente con el objeto de conocimiento correspondiente a cada una de las asignaturas del Plan de estudios.

Para propiciar esta acción de los estudiantes podemos recurrir a las técnicas didácticas: exposición, interrogatorio, entrevistas, visitas guiadas, discusiones en pequeños grupos, sociodramas, demostraciones, elaboración de mapas conceptuales, observaciones dirigidas, lecturas comentadas etc. y a los recursos auxiliares o apoyos didácticos: libros, apuntes, revistas, carteles, periódico mural, rotafolio, diapositivas, teatro guiñol, pizarrón, láminas, gráficas, películas, ilustraciones, modelos, videos, programas de televisión, videoconferencias, foros, chat, presentaciones en power point, consulta de bases de datos en la que destaca BIDI-UNAM (Biblioteca digital de la UNAM), de revistas electrónicas, de sitios especializados en la web y el uso de los motores de búsqueda como Google, Yahoo, etc.

La selección y organización de las técnicas y recursos didácticos forman las estrategias de enseñanza* que tendrán la intención de guiar a los estudiantes en su proceso de aprendizaje. Es decir, se seleccionarán con base en los objetivos de aprendizaje y con la intención de apoyarlos en la construcción de sentido y significado de los con- 
tenidos curriculares y de promover el autoaprendizaje (aprender a aprender) así como de "aprender a pensar". El uso de las nuevas tecnologías tendrá que incorporarse porque es un hecho que la escuela no debe estar al margen de ellas, pero tiene que fomentar su uso de una manera crítica y humana, no considerar que éstas son la panacea para resolver el problema del aprendizaje en la escuela, pero sí reconocer que son recursos que pueden enriquecer el proceso de enseñanza aprendizaje. ${ }^{4}$

Así como nadie puede alimentarse por otra persona, nadie puede aprender por otro. Cada persona va construyendo sus propios esquemas, como ya lo mencionamos anteriormente, de acuerdo a las experiencias, acciones o actividades que ella misma realiza. Por tal motivo la "exposición magistral", como único recurso para lograr que los alumnos aprendan, en muchas ocasiones es insuficiente. Se considera que con esta técnica los estudiantes tienen una actitud puramente receptiva, que no favorece la participación, propicia aprendizajes superficiales basados en la memorización. Sin embargo, sobre la exposición se pueden señalar ventajas e inconvenientes. ${ }^{5}$ Entre las primeras tenemos que, a través de esta técnica, se puede hacer una presentación de los contenidos temáticos para irlos conectando con los conocimientos previos de los estudiantes e ir reforzando los aspectos que ofrezcan alguna dificultad o confusión. Permite además, hacer síntesis o recapitulaciones que apoyen en la integración del conocimiento y puntualicen los aspectos más importantes. Esto implica una gran habilidad del docente para comu- nicarse y seguir el proceso grupal

Entre los inconvenientes de las clases magistrales, señalaremos que pueden llegar a convertirse en la única forma de relacionar a los alumnos con el conocimiento y por tanto se constituyen en una forma monótona, carente de interés, que corre el riesgo de llegar a ser dogmática y establecer una relación autoritaria a través de una comunicación unidireccional, con todos los riesgos que esto conlleva.

Lo más conveniente sería combinar formas de trabajo que incluyeran:

a) La participación del

docente en lo que a él le corresponde: organizar el trabajo, proporcionar la información pertinente en el momento oportuno (para centrar al grupo, para aclarar y ampliar información, para señalar y superar errores conceptuales, etc.), establecer los puentes cognitivos entre los conocimientos previos y los nuevos, para propiciar la construcción de aprendizajes significativos. Moderar las sesiones, observar y seguir el proceso grupal y apoyar en todo momento tanto individual como grupalmente.

b) Participación individual o autónoma. Para ello es necesario que los alumnos estudien tanto en sus apuntes, como en otras fuentes (pueden ser sugeridas por el docente, o bien por los mismos alumnos) y así desarrollar habilidades inherentes a este proceso (buscar y manejar bibliografía, seleccionar e integrar información, conocer y comparar diferentes enfoques teóricos, etc.). Así irán construyendo su estructura cognitiva que se enriquecerá en el intercambio con sus compañeros en actividades propuestas por el docente.

c) Actividades grupales en sus diversas modalidades: por parejas, pequeños grupos, sesiones plenarias, etc., que propicien la interacción de todo el grupo en una red de relaciones donde cada quien aporte sus conocimientos y experiencias, lo que los enriquecerá mutuamente

Así, es claro que las sesiones de clase no podrán ser monótonas. Las formas de trabajo variarán en cada sesión dependiendo de los contenidos, los objetivos y la organización de las estrategias de enseñanza y aprendizaje planeadas o modificadas según el proceso grupal. Con esto se puede intentar hacer del aprendizaje una actividad placentera y gratificante que propicie el crecimiento de los jóvenes. Esto es fundamental porque de ello depende un proceso en donde se construyen los vínculos: a) de los alumnos con el conocimiento, b) de los estudiantes con el profesor y c) entre los estudiantes que conforman el grupo escolar.

\section{SObRe LOS ObJetivos DE APRENDIZAJE}

Los objetivos constituyen la finalidad, (la tarea) de cada uno de los cursos, seminarios, talleres, etc. que conforman el programa de estudios (mapa curricular). Se relacionan con las capacidades o competencias que se pretende desarrollar en los estudiantes, referidos a los contenidos temáticos específicos.

Serán planteados como hipótesis, es decir; serán las propuestas de 
aprendizajes a lograr por los estudiantes al final del curso, seminario o taller a partir de las experiencias de aprendizaje vividas durante el proceso de enseñanza-aprendizaje.

La redacción de los objetivos se desprenderá del análisis de los siguientes aspectos:

- Los objetivos generales del currículum formal,

- El perfil del egresado y por tanto de los aportes de la materia al futuro profesional.

Para elaborarlos se sugieren los siguientes criterios:

a) Redactarlos en términos de conductas o aprendizajes que se pretende alcancen los estudiantes y que rebasen los niveles de la simple memorización o reproducción de información. Esto es, se propondrá más bien el logro de aprendizajes más complejos como son el desarrollo de capacidades intelectuales, por ejemplo; la observación, la comparación, la generalización, el análisis, la inferencia, la deducción, la síntesis, la crítica, la capacidad de plantear hipótesis o soluciones a diferentes problemas, pero sobre todo la de plantear nuevos problemas y tomar decisiones fundamentadas. Todo ello contribuye al desarrollo del pensamiento creativo o divergente. Esto le proporcionará armas al estudiante para enfrentar los retos que plantea una sociedad en constante cambio por los avances vertiginosos de la ciencia y la tecnología, así como los retos propios de una práctica profesional concreta (aprendizaje significativo.)

b) Plantearlos considerando las características generales de los grupos concretos, como son: edad, naturaleza de la asignatura, intereses, expectativas acerca de la materia, antecedentes académicos, perfil de egreso, etc.

c) Tener presente que los objetivos de un curso se refieren a conductas complejas, más totales, que abarquen o impliquen varias competencias, $\bullet$ por ejemplo se podría plantear que al final de un curso de Desarrollo de la personalidad infantil el objetivo de aprendizaje fuera el siguiente: Los alumnos analizarán las características del niño en sus diferentes etapas de desarrollo y detectarán las alteraciones de la personalidad más frecuentes para poder proporcionar orientación sobre el fomento a la salud mental. Esto implica que los estudiantes necesitan conocimientos sobre dinámica familiar, desarrollo psicológico durante los

- Se refiere a aquello que caracteriza a una persona capaz de realizar una tarea específica de manera eficiente, en un contexto determinado. Este término "es una consecuencia de la necesidad de superar una enseñanza que, en la mayoría de los casos, se ha reducido al aprendizaje memorístico de conocimientos, hecho que conlleva la dificultad para que éstos puedan ser aplicados en la vida real." Antoni Zabala, Idea clave 1, p.19

primeros años de vida, causas más frecuentes que alteran el desarrollo psicológico del niño, entre otros. Por otro lado, deben tener habilidades para proporcionar orientación psicológica a la familia, a la mujer embarazada y al niño; e identificar alteraciones de conducta en el niño, etc. Además deben desarrollar actitudes de respeto hacia los valores culturales de la familia, hacia el niño como persona; de interés por enfrentar los problemas psicológicos detectados en los diferentes entornos que afectan el desarrollo de la personalidad infantil, etc. En estas manifestaciones estarían involucrados valores como la responsabilidad, la honestidad, el compromiso, el amor a la profesión, etc., el objetivo incluye lo que hay que saber (conocimientos), saber hacer (procedimientos) y lo relacionado al ser (actitudes), es decir el objetivo hace alusión a diferentes competencias, tendiente a una formación integral.

Implícito en estos tres criterios estaría lo referente a los contenidos programáticos. Las conductas o aprendizajes que se pretende alcancen los estudiantes obviamente estarán relacionadas con los contenidos propios de cada materia. Algunos profesores consideran que para poder acreditar una materia los estudiantes deben "dominar" todo, o la mayor parte del programa. Quizá lo único que se les está pidiendo es que memoricen o reproduzcan información de los tex- 
tos, o los que el profesor ha "volcado" en sus disertaciones cotidianas (datos, fechas, nombres, clasificaciones, definiciones, etc. es decir, contenidos factuales). $\cdots$

Luego, hay que estar conscientes del tipo y nivel de aprendizajes o competencias que se plantean en relación con los contenidos, tales como: interpretar una teoría; analizar dos o más teorías y señalar sus diferencias epistemológicas y metodológicas; predecir un proceso o suceso a partir de una serie de elementos estudiados; analizar un modelo teórico y señalar sus implicaciones ideológicas; proponer alternativas de solución ante un problema detectado en la práctica; argumentar sobre la consistencia de un determinado enfoque teórico, etc.

Es necesario enfatizar, que los objetivos de aprendizaje son los logros que se pretende alcancen los estudiantes después de haber vivido una determinada estrategia de enseñanza-aprendizaje propuesta por el docente. Ejemplos de objetivos de aprendizaje:

- Contexto: Para una sesión de dos horas, en un curso de formación docente, con el tema "Motivación y aprendizaje" el objetivo puede ser:

Al final de la sesión los participantes explicarán la importancia que tiene para la práctica docente distinguir entre la motivación intrínseca y la extrínseca, referida tanto a los docentes como a los estudian-

- - Conocimiento de datos específicos: fechas, nombres, hechos, acontecimientos, situaciones, códigos, símbolos, clasificaciones, etc. cuyo aprendizaje requiere su memorización tes (contenido conceptual y actitudinal)

- Contexto: En un curso de Ética para la unidad "Algunos sistemas éticos" el objetivo de aprendizaje podría ser:

Al final de la unidad los estudiantes analizarán los diferentes sistemas éticos para identificar en cuál se ubica el que ellos sustentan y señalarán los comentarios que harían al respecto (contenido conceptual y actitudinal)

- Contexto: En un curso sobre "Contaminación ambiental" el objetivo terminal podría ser:

Al final del curso, los estudiantes elaborarán un proyecto para emprender acciones viables que permitan evitar o disminuir la contaminación ambiental en el hogar, en la escuela, en la oficina, en la fábrica, etc. dependiendo de su área de influencia (contenido conceptual, procedimental y actitudinal).

\section{Contenidos}

Generalmente los contenidos aluden al conocimiento de datos, conceptos, principios, teorías, leyes, teoremas,

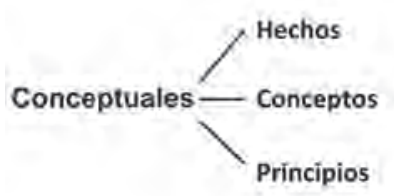

clasificaciones, procedimientos, etc., incluidos en los temarios escolares de cada materia o asignatura. Y en ocasiones la máxima preocupación de los docentes es cubrir o terminar el programa, sin preguntarse qué tanto han aprendido significativamente los estudiantes.

Actualmente este término tiene un significado más amplio. Es todo aquello que hay que aprender para alcanzar los objetivos de aprendizaje, que no sólo incluyen capacidades cognitivas, sino que incluyen todas las capacidades inherentes al ser humano, como las psicomotrices, afectivas, de relación, etc.

César Coll ${ }^{1-3}$ agrupa los contenidos en: conceptuales (qué hay que saber), procedimentales (qué hay que saber hacer) y actitudinales (cómo hay que ser), en esta propuesta subyace una tendencia a una formación integral de los estudiantes.

Esta clasificación se propone con fines de análisis, ya que en la realidad la conducta humana no está disociada. Cualquier comportamiento calificado de eficaz o competente implica elementos tanto conceptuales, como procedimentales, así como actitudina-
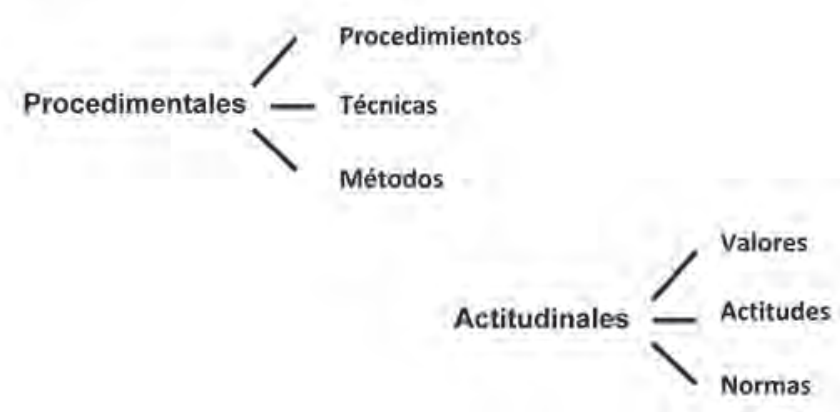
les. Ser competente significa poseer conocimientos (factuales y conceptuales), que no sirven de nada si no se sabe manejarlos, utilizarlos mediante ciertos procedimientos (estrategias, métodos, procedimientos, técnicas, etc.), así como reflexionar sobre su aplicación, sus posibles implicaciones éticas, su impacto social, etc. es decir, elementos actitudinales. Así, toda actuación considerada como competente implica el dominio de conocimientos vinculados con habilidades y actitudes.

\section{Metodología del proceso ENSEÑANZA-APRENDIZAJE}

Uno de los problemas que enfrenta la educación escolarizada es el de lograr una participación más significativa por parte de los estudiantes en el proceso de aprendizaje. Esto implica una responsabilidad muy concreta e insoslayable del profesor.

Lo primero que hay que aclarar es que esa participación de los alumnos no se refiere al hecho de que sean ellos los que preparen y presenten al grupo "los temas", individualmente o en equipo. Por razón obvia esto es muy cuestionable, en primer lugar porque ellos no tienen la preparación necesaria para poder discriminar, integrar, relacionar, una serie de conceptos relevantes; no conocen la estructura de la disciplina. Generalmente se concretan a leer información o presentar láminas, esquemas, etc., en forma desarticulada y descontextualizada con la finalidad de cumplir con la tarea y obtener una calificación.

Tampoco podemos seguir pensando en el docente que va a "exponer la clase". Si convenimos en que el aprendizaje es un cambio en la forma de actuar, pensar y sentir de un individuo como resultado de su experiencia, podemos descartar la posibilidad de que el alumno aprenda, si sólo se concreta a contemplar la actuación del docente.

Carl Rogers ${ }^{6}$ afirma que la experiencia es intransferible, es decir, que la experiencia es un mundo privado para cada individuo; en un sentido popular "nadie experimenta en cabeza ajena". Nadie puede aprender por otros y transmitir o regalar un aprendizaje. El aprendizaje es un asunto personal e íntimo.

Dentro de las actividades de aprendizaje no podemos omitir el papel que le corresponde al docente desde el punto de vista del constructivismo, que califica la enseñanza como una "ayuda ajustada" al proceso de aprendizaje, porque ésta no puede sustituir la actividad mental de construcción del alumno ni ocupar su lugar ${ }^{7} \mathrm{El}$ aprendizaje es un proceso activo por parte del alumno, durante el cual construye, modifica, enriquece y diversifica sus esquemas de conocimiento, a partir del sentido y significado que puede atribuir a los contenidos escolares. ${ }^{7}$

Al preparar, planear o diseñar un curso, el profesor es el que selecciona y construye los objetivos de aprendizaje, investiga sobre los contenidos, los analiza, organiza, relaciona, prepara material de apoyo, expone y evalúa los resultados. Si aceptamos que la educación, como la gimnasia en la que sólo se benefician de ella quienes la practican, podemos explicarnos por qué los profesores aprenden tanto dando clases.

Lo que los profesores hacen en el momento de "dar la clase", es dictar su conclusión, su síntesis, el producto de su empeño y de su estudio, olvidando el proceso que fue necesario para llegar a esta claridad de ideas. Para que los alumnos lleguen a lograr los mismos aprendizajes y capacidades del profesor tendrían que pasar por las mismas experiencias u otras de naturaleza semejante.

Puede considerarse que una actividad de aprendizaje es una tarea concreta, por medio de la cual el estudiante se pone en contacto con el objeto de estudio o situación de aprendizaje. Las actividades de aprendizaje son las generadoras de los cambios, es decir; de la experiencia que se desea ocurra en los alumnos. Las actividades pueden ser directamente controladas por el profesor, no así las experiencias. A través de la experiencia "las personas cambian su manera de ver las cosas, enriquecen la forma de mirarse a sí mismas, a los otros y a la realidad que los rodea".

Sólo hay aprendizaje cuando hay experiencia, una actividad por el simple hecho de realizarla no siempre produce experiencia. La actividad en sí no es más que una serie de movimientos, pasos y mecanizaciones, que sin contacto con la conciencia se actúa sin trascender a un aprendizaje significativo

Así, las actividades de aprendizaje deben propiciar la experiencia, procurando la intervención activa del que aprende, es él quien asimila lo que él mismo hace, no lo que hace el profesor. La participación, la interacción con sus compañeros y con el docente le brindarán la oportunidad de construir aprendizajes significativos.

Es en la búsqueda de experiencias de aprendizaje donde el docente tiene que aportar su máxima creatividad, haciendo un análisis de las actividades propuestas. Esto con el fin de corroborar si tienen relación con los alumnos, si los involucra 
personalmente, si son retos accesibles que activan sus motivaciones y si concuerdan con sus necesidades, capacidades y posibilidades.

En la organización de las actividades de aprendizaje, los docentes cuidarán que unas actividades las realicen ellos y otras los alumnos ya sea individualmente o en pequeños grupos. Así el docente no será el único actor del proceso y los estudiantes meros espectadores. Con esto se busca establecer un equilibrio en las actividades para que tanto profesores como alumnos jueguen un papel protagónico.

El docente tiene que desempeñar diferentes funciones; como coordinador, informador, etc., pero también como promotor de la interacción grupal, de la comunicación y como observador y seguidor del proceso grupal. Esto propicia que se cree un clima adecuado para el aprendizaje y que se vaya aprovechando y cuidando el proceso grupal (momentos de interés, desinterés, producción, obstáculos, conflictos, ansiedades, cansancio, etc.). No se tratará de seguir al pie de la letra lo planeado, sino de hacer los ajustes necesarios y dirigir el proceso grupal que es impredecible. Estamos hablando de una relación pedagógica más flexible, democrática, humana, donde el profesor aprende en la medida que enseña y los estudiantes enseñan en la medida que aprenden. ${ }^{9}$ En esta relación el profesor va siguiendo el proceso grupal y va dándole dirección, en un compromiso compartido con los estudiantes, lo que rompe con la práctica habitual en la que el grupo se adapta y acepta las condiciones impuestas por el profesor en una relación autoritaria y despersonalizada.
Algunos criterios para seleccionar actividades de aprendizaje.

- Que sean significativas para los estudiantes. Esto es, tiene que haber una relación con sus necesidades, intereses, inquietudes y experiencias de vida y que contribuyan a construir significados y atribuir sentido a lo que hacen. En esa medida les ayudarán a crecer personal y profesionalmente.

- Que favorezcan la vinculación de los nuevos contenidos con los conocimientos previos de los estudiantes. Para ello es conveniente establecer diálogos entre el docente y los estudiantes, así como elaborar resúmenes, mapas conceptuales al inicio del curso o de una unidad que confrontarán al final para enriquecerlos, etc.

- Que las actividades propuestas tiendan a provocar un conflicto cognitivo que desencadene la actividad mental de los estudiantes e influyan en una actitud favorable para el aprendizaje.

- Que propicien en el alumno la oportunidad de alcanzar los aprendizajes propuestos en los objetivos.

- Que estén dentro de las posibilidades de los alumnos para realizarlas, en cuanto a las condiciones, niveles de conocimiento y las habilidades necesarias (retos abordables).

- Que sean variadas; sobre los mismos contenidos proponer diferentes actividades para que tengan la libertad de seleccionar cualquiera: investigar, recabar información sobre actualidades científicas en libros, revistas, bases de datos, revistas electrónicas, etc.; presentar un proyecto, hacer entrevistas, cuestionarios, elaborar materiales escritos, modelos, maquetas, gráficas, videos, etc. El profesor organiza la presentación coherente de las actividades seleccionadas individualmente o en equipo.

- Que propicien la participación e interacción de todos los miembros del grupo, para dar oportunidad a la confrontación de lo que piensan acerca de una temática, problema o concepto concreto. Esto generará aprendizajes colaterales como por ejemplo: aprender a escuchar, ser tolerantes frente a puntos de vista diferentes a los propios, argumentar y fundamentar sus puntos de vista, reconocer que pueden estar equivocados, que existen diferentes formas de enfocar y resolver un problema, que el conocimiento es un proceso permanente y que no es neutro, que tiene implicaciones sociales, éticas, políticas, así como muchas otras.

- Que promuevan la creación de Zonas de Desarrollo Próximo (ZDP) que Vigotsky define como "la distancia entre el nivel real de desarrollo, determinado por la capacidad de resolver independientemente un problema, y el nivel de desarrollo potencial, determinado a través de la resolución de un problema bajo la guía de un adulto o en colaboración con otro compañero más capaz."10 Es decir, cuidar que en el 
desarrollo de una unidad didáctica, las actividades promuevan la interacción grupal, mediante actividades en pequeños grupos o plenarios que puedan desencadenar procesos de construcción, modificación, enriquecimiento y diversificación de los esquemas de conocimiento. ${ }^{7}$ También se debe fomentar la discusión, la confrontación de esquemas, para abrir la posibilidad de ampliar y consolidar los aprendizajes que se construyeron individualmente

- Que la diversidad de actividades tienda a promover el desarrollo de competencias actitudinales, conceptuales y procedimentales en los estudiantes, para que los conduzcan a ser más autónomos en su aprendizaje (aprender a aprender).

- Entre algunos de los aspectos trascendentes de la selección de las actividades de aprendizaje está el impacto que éstas pueden tener no sólo en la construcción de conocimientos, sino también su repercusión en el autoconcepto y autoestima de los estudiantes. Esto se logra de manera positiva mientras sean actividades que ellos puedan realizar, en conclusión, que estén dentro de sus intereses, que sean gratificantes, en tanto les permitan darse cuenta de sus posibilidades y competencias, (retos abordables). Esto permitirá que los jóvenes tengan un desarrollo de su personalidad más pleno, lo que les dará mayor seguridad, madurez intelectual y emocional, más independencia y autodeterminación, así como conciencia de la necesidad de aprender a convivir de una manera más sana.

Por tanto, contenidos y método son parte de un mismo proceso. No sólo se aprenden contenidos, sino formas de relacionarse con ellos, formas de pensar, de sentir la realidad y de actuar frente a ella, y además formas de establecer vínculos con los demás seres humanos.

\section{Evaluación DE LOS APRENDIZAJES}

Empezaremos por distinguir los conceptos de evaluación y acreditación porque no son sinónimos. El primero es más amplio e incluye al segundo. Evaluar es valorar de manera crítica y autocrítica una acción o proceso a partir de un análisis minucioso realizado por todas las personas involucradas. El objetivo sería mejorar, adecuar y transformar lo que se reconoce como necesario para que con base en la experiencia vivida se hagan los ajustes pertinentes, además de recuperar aquello que se considera adecuado o valioso. En coherencia con la idea anterior, no sólo tenemos que apreciar los logros de los estudiantes al final del proceso, sino también analizar la estructura del programa de estudios, la metodología de enseñanza- aprendizaje, los materiales empleados, la bibliografía, los objetivos, la metodología de trabajo y por tanto las actividades de aprendizaje, los ejemplos utilizados, el vocabulario empleado, el papel del docente, las condiciones para el estudio de los

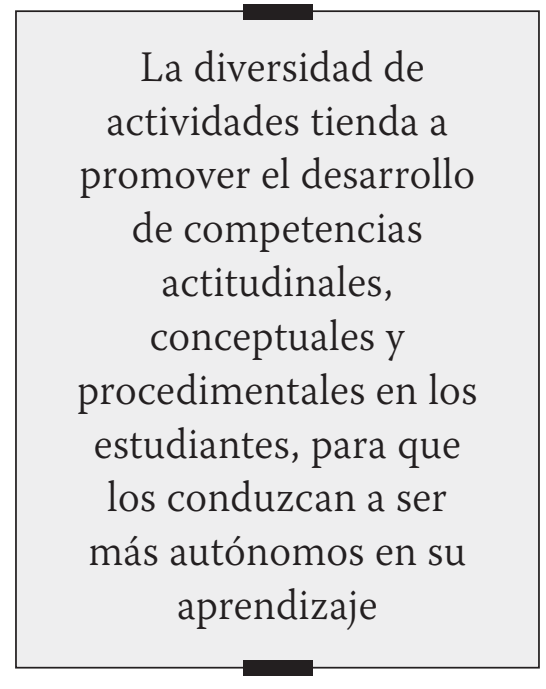

participantes, el tiempo asignado a cada asignatura, etc. Es necesario reconocer que en este proceso de análisis tienen que participar tanto docentes como estudiantes para poder recabar información más objetiva que permita ampliar la evaluación a través de las miradas de todos los que vivieron la experiencia. De esta manera, se puede contar con elementos que fundamenten los ajustes o cambios necesarios, así como el reconocimiento de los aspectos positivos del proceso y de los logros alcanzados.

La autoevaluación es otro ejercicio necesario para que los estudiantes tengan conocimiento de los aprendizajes logrados, tanto como de sus avances y sus deficiencias, o bien aquello en lo que tienen que poner atención para superarlo, ya que es necesario para su desarrollo personal y profesional. Para realizar este ejercicio el docente señalará algunos criterios, como pueden ser: la asistencia y puntualidad, el compromiso con el grupo y con la tarea, si se realizaron las actividades extraclase, si prepararon y participaron en las actividades grupales, si tienen sentido de pertenencia al grupo, y por último que señalen los apren- 
dizajes significativos logrados en el curso así como los obstáculos enfrentados, etc.

\section{Acreditación}

Una de las acepciones de acreditar es "dar pruebas", ¿de qué se necesita tener pruebas en el proceso de enseñanza-aprendizaje? Lo que se buscaría probar es que los estudiantes dominan los aprendizajes mínimos necesarios, de cada una de las asignaturas del plan de estudios, para realizar una práctica profesional determinada. Estos aprendizajes son los que se expresan en los objetivos terminales o generales que se plantean en cada curso, seminario o taller y se basan en las competencias de conocimientos, habilidades y actitudes, que deben desarrollar los estudiantes en cada una de las materias del mapa curricular.

Ahora bien, esta acreditación también necesita planearse. Para esto se tendrá que revisar la coherencia entre objetivos, contenidos y actividades de aprendizaje, para analizar si tales contenidos y estrategias de enseñanza propiciaron el logro de los objetivos de aprendizaje, tanto del curso en su totalidad, como de las unidades temáticas en particular y precisar los aportes que cada asignatura hace a la conformación del perfil del egresado.

Las evidencias del logro de aprendizajes significativos no necesariamente se refieren a la aplicación de las llamadas "pruebas objetivas"...' Si somos consecuentes con un concepto de educación y aprendizaje que implica conductas complejas, que involucran al sujeto en su totalidad, que se refieren a la capacidad de tomar decisiones, de argumentar, de proponer, de plantear problemas y soluciones, de utilizar el conocimiento en situaciones concretas, es decir de desarrollar su "capacidad de pensar", estaremos de acuerdo en que no sólo las pruebas ya mencionadas son los únicos instrumentos posibles para comprobar el tipo y nivel de objetivos alcanzados. Podemos pensar en otras posibilidades, como por ejemplo realizar un ensayo con especificaciones previas sobre el contenido, una investigación bibliográfica, la propuesta de un plan o proyecto, trabajos que impliquen diferentes actividades como entrevistas, análisis estadísticos, comparación de diferentes grupos o enfoques, elaboración de mapas conceptuales, etc. Actualmente podemos incluso pensar en el uso de algunos medios interactivos en línea, como la elaboración de un blog, de una página web o bien diferentes actividades que ofertan los software educativos.

Es decir, se pensaría en situaciones que permitan tanto al docente como a los propios estudiantes apreciar las competencias que se desarrollaron, en cuanto al dominio y uso del conocimiento, así como el desarrollo de ciertas habilidades intelectuales y psicomotrices, los valores y actitudes implicados, así como su vinculación con el perfil profesional.

\footnotetext{
.... Son las que se aplican comúnmente en las escuelas (opción múltiple, correspondencia de coIumnas, ordenamiento cronológico, respuesta breve, falso y verdadero, etc.), cuya objetividad está cuestionada actualmente. Son exámenes escritos que intentan eliminar en la medida de lo posible, la subjetividad del evaluador al analizar, procesar y calificar dicha prueba.
}

\section{ReferenCIAS BIBLIOGRÁFICAS}

1 Zabala A. La práctica educativa. Cómo enseñar. Barcelona: Graó 1998.

2 Koplowitz, H. La epistemología constructivista de Piaget. Exploración y comparación con varias alternativas teóricas. En: C Coll (ed). Psicología genética y educación. Barcelona: Oikos-Tau, 1981.

3 Coll C. Significado y sentido en el aprendizaje escolar. Reflexiones en torno al concepto de aprendizaje significativo. En: Coll C. Aprendizaje escolar y construcción del conocimiento. Argentina: PaisosEducador,1990.

4. Fernández M, Fernández N. Bases para la formación del pensamiento crítico del profesor para las nuevas tecnologías. [Consultado el 8 de marzo de 2010]. Disponible en:

http:/www.sol.edu/portal/modules. php?name $=$ News\&file $=$ print $\&$ sid $=10$

5 Zabalza MA. Competencias docentes del profesorado universitario. Calidad y desarrollo profesional. Madrid: Narcea, 2003.

6. Rogers C. El proceso de convertirse en persona: mi técnica terapéutica. México: Paidos; 1993.

7 Onrubia J. Enseñar: crear Zonas de Desarrollo Próximo e intervenir en ellas. En: Coll C, Martín E, Mauri T, Miras M, Onrubia J, Solé I, Zabala A. El constructivismo en el aula. Barcelona: Graó, 1999.

8 Quiroga AP. Coord. El proceso educativo según Paulo Freire y Enrique Pichon-Rivière. México: Plaza y Valdés,1998.

9 Freire P. Pedagogía del Oprimido. 8a.edición. México: Siglo XXI; 1973.

10 Baquero R. Vigotsky y el aprendizaje escolar. Argentina: Aique; 1999. 